\title{
A Reflection Upon Speed Conference at the "Theoretical Archaeology Group (TAG) Conference", Hosted by the Department of Archaeology,University of Glasgow, $17^{\text {th }}$ $19^{\text {th }}$ December 2004
}

\author{
James Doeser \\ Institute of Archaeology, UCL
}

"Tartan TAG", the most recent "Theoretical Archaeology Group Conference", took place in Glasgow in December 2004. Amongst the usual collection of sessions on epistemology, materiality, post-structuralism and other contemporary preoccupations of an increasingly self-aware academic discipline, there nestled a session whose aim was simple yet ambitious, and which may have been the most important session of the whole conference.

The presentation of papers at archaeological conferences may be entertaining, interesting and enlightening. These qualities (or lack thereof) will depend upon the character of the speaker, the nature of their work and a myriad of other factors (such as the time of day, level of what may be called 'conference-fatigue', etc.). Like the organisers of Speed Conference, I have often found the real value of conferences to lie in the discussion periods at the ends of sessions or during the informal (but no less important) social events.

The conventional 20-minute paper has much in its favour: it allows the presenter to make two or three basic assertions which may then be illustrated with practical examples or further theoretical elaboration. Time for discussion is usually scheduled at the end of papers or sessions. Often, however, this is sacrificed to overrunning papers or becomes dominated by two or three individuals with their own agendas to push.

As the session organisers of Speed Conference (James Dixon, Pre-Construct Archaeology; Sam Hardy, University of Sussex; Thomas Kador and Jane Ruffino, University of Dublin) state in their abstract, "conferences appear to be about dialogue and, in turn, this dialogue should drive our discipline forward". Personally, I always use conferences as a means to air my own speculative ideas, seeking either reassuring agreement or intellectual destruction. Airing uncertainties and suspicions at the frontiers of our own research, leaving ourselves open to attack, is vital to achieving the desired 'driving forward' of our discipline. Speed Conference left behind the 20-minute paper and brought discussion to the fore. It achieved a new level of discourse in the most constructive and interesting of ways.

The format of Speed Conference was derived from the widespread phenomenon of Speed Dating, and worked as follows: 
The session was divided into two parts. The format of part one matched that found at a Speed Dating event. Each participant (attendee or organiser) in the session was paired with another for a one-to-one discussion round lasting five minutes. The topic of conversation in each round was based on the theme of "Archaeology and Time". At the end of each round, "time!" was called by the compere, and every participant wrote down a couple of lines on a Post-it note. These musings were usually (though not necessarily) inspired by the preceding conversation, and the notes were then stuck onto a whiteboard. Participants proceeded to form new pairs and the next round began. This process continued until every combination of people had been paired (there were about 12 of us in total), and the whiteboard was well-adorned with Post-it scribbles.

Part two of Speed Conference then took place. The discussant (in this case Angela Piccini, University of Bristol) arranged our assorted jottings and instigated a roundtable discussion inspired by some of the recorded comments. As one might expect when grappling with a subject like archaeology and time, the discussion moved in numerous directions. Individual philosophies jostled against one another as participants, their confidence increased and thoughts refined by the previous face-to-face encounters, keenly engaged in pushing the boundaries of contemporary archaeological frameworks. Suspicions were aired and questions asked in an atmosphere of mutual adventure. Whenever a philosophical cul-de-sac was encountered, the discussant moved things along with another Post-it-inspired question.

Having since read the abstract and session aims in the TAG Handbook, I see that it was expected of Speed Conference participants to have read (or thought) about the subject of time prior to coming to the session. I did nothing of the sort, arriving totally unprepared, wandering in after leaving another session whose post-coffee-break papers were of little interest to me. This prerequisite may have contributed to the relatively low attendance at the session, though this was more likely due to interest in other sessions. My unpreparedness did not impair my participation in the session and, had I been aware of what was expected of me, I still would have come unprepared. TAG sessions can be taxing enough without the voluntary undertaking of homework!

In general, the session was a good idea and well executed. However, there are a number of residual problems. As with all conference sessions, the relative productivity depends upon who is present and contributing. In this instance the session was attended by some confident and articulate participants, yet this might not necessarily have been the case. Although the direction of the session was somewhat 'off the leash', this may have been a good thing: conflicts and corroborations between participants could not be engineered in the same fashion as they might have been by organisers of conventional conference sessions.

The fundamental drawback of this session format, however, was the difficulty in arriving at a satisfactory end-product. The traditional format of presentations followed by discussion can lead to a general understanding of what most speakers thought, and of how this may be of use, or a source of concern, or perhaps even totally boring, to session attendees. This is not always the case: conference sessions can lead in no di- 
rection whatsoever, or in a disappointingly consensual and straightforward direction. Whichever course the traditional format session takes, it also lends itself to publication through the edited volume (occasionally with a discussion chapter included).

The innovative Speed Conference format is in its infancy, and a solution to the problem of leaving a permanent legacy of such sessions may yet emerge. Like its real-world equivalent of Speed Dating, Speed Conference was a lot of fun, and although, as far as I am aware, no romances were initiated in the Glasgow session, there is a growing recognition that there are alternatives to stagnant and often unsatisfactory conference formats. I hope that my fellow participants appreciated the opportunity to put dialogue centre stage. 